\title{
Optimal configuration of distributed generation based on improved fruit fly optimization algorithm
}

\author{
Pan Chao ${ }^{\mathrm{a}}$, Lv Jiahui ${ }^{\mathrm{a}}$, Meng Tao ${ }^{\mathrm{b}}$ \\ ${ }^{a}$ School of Electrical Engineering, Northeast Dianli University, Jilin 132012, China \\ ${ }^{b}$ Electrical Power Research Institute, State Grid Jilin Electric Power Co.Ltd, Changchun 130001, China
}

\begin{abstract}
Optimal configuration of distributed generation in power distribution network is researched in this paper. Considering investment benefit, voltage quality and power loss of system, and a multi-objective optimal configuration model is established with fuzzy technique, which could efficiently solve the excessive optimization problem for different dimension of targets. A new bionic intelligence algorithm-fruit fly optimization algorithm is improved and the operation of attraction and repulsion is introduced into this algorithm by learning the chemotaxis of bacteria in foraging process to improve the population diversity and reduce the probability of falling into local optimization. Simulation results of IEEE33 node system demonstrated that, compared with the traditional fruit fly optimization algorithm and particle swarm optimization algorithm, improved fruit fly optimization algorithm has great advantage in optimization speed and solution accuracy, and is able to search the optimal configuration rapidly and effectively, which verify the validity and reasonability of this improved algorithm.
\end{abstract}

Keywords: Fruit fly optimization algorithm, power distribution network, distributed generation, multi-objective optimization, comprehensive membership degree

\section{Introduction}

Distributed Generation (DG) as a significant component of future energy strategy structure has received extensive attention in domestic and foreign. With large-scale of DG access to the distribution network, the operation complexity and uncertainty of distribution system is increased. Reasonable optimal configuration will help to reduce power loss of system, improve the voltage quality and enhance load rate. On the contrary, it will be counterproductive or even cause a serious of problems like deterioration of voltage quality, reverse power and decrease of system security. Consequently, it is practical and significant to research the optimal configuration of DG in power distributed network.

The study of optimal configuration of power distributed network with DG has become a hot issue. A bi-level programming model is established in literature [1], and the chance constraint method is used to solve the installation location and capacity of DG and capacitor bank. In literature [2], the factors of demand-side response is blended into the optimal configuration model of DG, and a bi-level programming model is built to analysis the benefits of power grid company, the voltage level and power losses of the system. Consider of DG unit investment profits and the benefits of improved power grid, a multi-objective optimal configuration model is established in literature [3], and the improved nondominated sorting genetic algorithm is used in solving the problem and searching for balance of interest between the power generation enterprises and the power supply companies.

Consider the factors of investment benefit, network loss and branch voltage stability margin, and a multi-objective programming model is established in this paper. Compared with the particle swarm algorithm and the traditional fruit fly optimization algorithm, a new bionic intelligence algorithm-fruit fly optimization algorithm is improved to solve this model, and the rationality of the model and the validity

Manuscript received April 15, 2016; revised October 14, 2016.

Corresponding author. Tel.: 861-594-865-1077; E-mail address: $2584273861 @ q q . c o m$.

doi: $10.12720 /$ sgce.5.4.237-244 
of the improved fruit fly optimization algorithm are verified by analyzing the simulation results of IEEE33 node power distribution system.

\section{Multi-objective Fuzzy Programming Model}

\subsection{Objective function}

2.1.1. Investment cost of $D G$

The investment gains of DG unit is [4]:

$$
C_{\mathrm{psc}}=\frac{C_{\mathrm{tpf}}}{C_{\mathrm{inv}}}
$$

where $C_{\mathrm{tpf}}$ is the annual translation gain with DG including electricity sales revenue of DG and Governments subsides, and $C_{\mathrm{inv}}$ is the investment cost of DG converted to each year which containing the cost of land, installation and the operating and maintenance cost.

$$
\begin{aligned}
& C_{\mathrm{tpf}}=8760 \sum_{i=1}^{M}\left(C_{\mathrm{gp} i}+C_{\mathrm{ap} i}\right) S_{i}^{\mathrm{rated}} \lambda_{\mathrm{cf} i} \\
& C_{\mathrm{inv}}=\sum_{i=1}^{M} \alpha_{\mathrm{dg} i} S_{i}^{\mathrm{rated}} C_{i}^{\mathrm{fixed}}+8760 \sum_{i=1}^{\mathrm{M}} C_{i}^{\mathrm{op}} S_{i}^{\mathrm{rated}} \lambda_{\mathrm{cf} i}
\end{aligned}
$$

where $C_{\mathrm{gp} i}, C_{\mathrm{ap} i}$ are the policy subsidy electricity price and the electric price in connection-grid, respectively. $M$ is the amount of different types of DG. $S_{i}^{\text {rated }}, C_{i}^{\text {fixed }}$ and $\alpha_{\mathrm{dg} i}$ are the conversion coefficient of the annual investment cost of the type of DG, unit investment cost of each year and the rated installation capacity. $C_{i o p}$ is the output fuel cost and operating and maintenance cost of the $i$ type of DG unit. $\lambda_{\mathrm{cf} i}$ is the Rated capacity factor of the $i$ type of DG.

2.1.2. Voltage index

Voltage index contains the branch voltage stability index and the index of node voltage deviation. The branch voltage stability index is the sum of all the branch voltage stability indices describing the voltage stability margin of all branches [5]. The voltage deviation index is the sum of the differences of all the node voltage and the reference voltage reflecting the offset of the whole node voltage. Both indices sufficiently measure the voltage quality of the power distribution network as:

$$
\begin{aligned}
& u_{1}=\sum_{i=1}^{N} \sum_{j=1}^{T}\left[4\left(P_{j} X_{i j}-Q_{j} R_{i j}\right)^{2}+4 U_{i}^{2}\left(P_{j} X_{i j}+Q_{j} R_{i j}\right)\right] / U_{i}^{4} \\
& u_{2}=\sum_{i=1}^{N}\left|U_{i}-U_{\text {rate }}\right|
\end{aligned}
$$

where $u_{1}$ is the branch voltage stability index and $u_{2}$ is the voltage offset index. $N$ is the number of network node. $T$ is the number of network load node. $P_{j}, Q_{j}$ are the active load and the reactive load of $j$ node. $R_{i j}, X_{i j}$ are the resistance and the reactance of the $i j$ branch.

Considering the node voltage offset index and the branch voltage stability, the evaluating indicator of voltage is:

$$
U=\alpha_{1} u_{1}+\alpha_{2} u_{2}
$$

where $\alpha_{1}, \alpha_{2}$ are the weighting coefficient of $u_{1}$ and $u_{2}$, both value as 0.5 . 


\subsubsection{Power loss}

Power loss is an important index to evaluate the economical operation of power distribution network. The reasonable optimal configuration of DG can reduce the power loss effectively, the expression of power loss is:

$$
P_{\text {loss }}=\sum_{k=1}^{L}\left|I_{k}\right|^{2} R_{k}
$$

where $L$ is the number of branch. $I_{k}$ and $R_{k}$ are the current and the resistant of the $k$ branch.

\subsubsection{Objective function}

According to the analysis above, the multi-objective programming problem can be translated into single one, as given in (8).

$$
\max F=a \lambda_{1}+b \lambda_{2}+c \lambda_{3}
$$

where $a, b, c$ are the weighting coefficient of $\lambda_{1}, \lambda_{2}, \lambda_{3}$, which all take the value of $1 / 3 . F$ is the integrated membership grade and the larger the value indicates that the planning scheme is more superior.

\subsection{Constraint condition}

The equality and inequality constrains of multi-objective programming of DG are:

$$
\left\{\begin{array}{l}
P_{i}-P_{\mathrm{DG} i}=\sum_{j=1}^{N o d e}\left[e_{i}\left(G_{i j} e_{j}-B_{i j} f_{j}\right)+f_{i}\left(G_{i j} f_{j}+B_{i j} e_{j}\right)\right] \\
Q_{i}-Q_{\mathrm{DG} i}=\sum_{j=1}^{N o d e}\left[e_{i}\left(G_{i j} e_{j}-B_{i j} f_{j}\right)-f_{i}\left(G_{i j} f_{j}+B_{i j} e_{j}\right)\right] \\
S_{\mathrm{DG}_{-} j} \leq S_{\mathrm{DG}_{j} j}^{\max }, i \in N \\
U_{i, \min } \leq U_{i} \leq U_{i, \max } \\
\sum_{i=1}^{N} P_{\mathrm{DG}_{-} i} \leq \delta \sum_{j=1}^{N_{\text {bus }}} P_{j} \\
I_{k} \leq I_{k \max }
\end{array}\right.
$$

where $P_{\mathrm{DG}}, Q_{\mathrm{DG} i}$ are the active and the reactive power of node $i . G_{i j}, B_{i j}$ are the conductivity and the susceptance of the $i j$ branch. $e_{i}, f_{j}$ are the real and the imaginary part of the node $i$ voltage. $I_{k \max }$ is the maximum current of the branch $k$ allowed. $U_{i, \max }, U_{i, \min }$ are the maximum and the minimum voltage of node $i$ allowed. $\delta$ is the limit coefficient of DG's permeability.

\section{Improved Fruit Fly Optimization Algorithm}

\subsection{Fruit fly optimization algorithm}

Fruit fly optimization algorithm (FOA) [6] is a new bionic intelligence algorithm proposed by following the Drosophila foraging behavior. Based on the characteristics of fruit fly in searching food, the implementation of fruit fly optimization algorithm can be described as:

- a) Set the population size (Sizepop) and the maximum iterations $\left(T_{\max }\right)$. Then initialize group position $X \_$axis and $Y \_$axis randomly within the scope of feasible region.

- b) Calculate the random searching direction and distance of individual fruit fly, as :

$$
\left\{\begin{array}{l}
X_{i}=X_{-} \text {axis }+ \text { RandValue } \\
Y_{i}=Y_{-} \text {axis }+ \text { RandValue }
\end{array}\right.
$$


where RandValue is the random searching distance.

- c) The distance between the individual of fruit fly and original spot $\left(D_{\text {isti }}\right)$ (the food place) is the unknown that is going to solve and the determination value of the individual odor concentration $(\mathrm{Si})$ can be calculated as:

$$
\left\{\begin{array}{l}
D_{\text {isti }}=\sqrt{X_{i}^{2}+Y_{i}^{2}} \\
S_{i}=1 / D_{\text {isti }}
\end{array}\right.
$$

- d) Put the determination value of every individual odor concentration into the function of odor concentration (or called objective function), and get the current value of the individual odor concentration, as follows:

$$
\operatorname{Smell}(i)=\operatorname{Function}\left(S_{i}\right)
$$

- e) Rank the individual odor concentration to find the individual with the best odorousness (Suitable for solving the minimum value problem), as:

$[$ bestSmell, bestindex $]=\min (\operatorname{Smell}(i))$

- f) The optimal odor concentration value and group position are recorded and saved as:

$\left\{\begin{array}{l}\text { Smellbest }=\text { bestSmell } \\ X_{-} \text {axis }=X(\text { bestindex }) \\ Y_{-} \text {axis }=Y(\text { bestindex })\end{array}\right.$

- g) Repeat as necessary for each additional affiliation. Repeat steps from a) to e) to execute iterative refinement. And step f) will be executed if the current iteration is less than the maximum and optimal odor concentration value in every iteration is better than the last one.

\subsection{Improved fruit fly optimization algorithm}

The chemotaxis thought in the foraging process of the bacteria that reject (away from the exterminator) and attract (near the attractant) operation is introduced into fruit fly optimization algorithm process to determine whether performing an exclusive operation or an attractive operation according to the variance of odor density, which adjusts group location update strategy dynamically in this paper.

Solving steps of improved fruit fly optimization configuration is:

- a) Parameter initialization.

- b) Initialize the group location as (10), and calculate the odor determination and the odor concentration of individuals as (11) and (12).

- c) Record and save the best and the worst odor concentration and its location of the global as (15).

$$
\left\{\begin{array}{l}
\text { Smellbest }=\text { bestSmell } \\
X_{b}=X(\text { bestindex }) \\
Y_{b}=Y(\text { bestindex }) \\
\text { Smellworst }=\text { worstSmell } \\
X_{w}=X(\text { Smellworst }) \\
Y_{w}=Y(\text { Smellworst })
\end{array}\right.
$$

- d) Calculate the average population odor concentration Smell $l_{\text {avg }}$ and its variance $\sigma^{2}$ as: 


$$
\begin{aligned}
& \text { Smell } l_{\text {avg }}=\sum_{i=1}^{\text {Sizepop }} \operatorname{Smell}(i) / \text { Sizepop } \\
& \sigma^{2}=\sum_{i=1}^{\text {Sizepop } \left._{\text {Sirell }}(i)-\text { Smell }_{\text {avg }}\right)^{2} / \text { Sizepop }}
\end{aligned}
$$

- e) Compare the variance with $\delta$ given as variance threshold. If $\sigma^{2} \leq \delta$, the exclusive operation as (18) will be executed, otherwise the attractive operation as (19).

$$
\begin{aligned}
& \left\{\begin{array}{l}
X_{-} \text {axis }=X_{-} \text {axis }-X_{w} \\
Y_{\text {_axis }}=Y_{\text {_axis }}-Y_{w}
\end{array}\right. \\
& \left\{\begin{array}{l}
X_{\text {_axis }}=X_{\text {_axis }}+X_{b} \\
Y_{\text {_axis }}=Y_{\text {_axis }}+Y_{b}
\end{array}\right.
\end{aligned}
$$

- f) Execute iterative optimization by repeating step b) and c) and step d) to e) will be repeated, if the current iteration is less than the maximum and the best odor concentration of every iteration is superior to the last.

The flow chart of solving the problem of DG multi-objective fuzzy programming based on the improved fruit fly optimization algorithm (MFOA) is shown below:

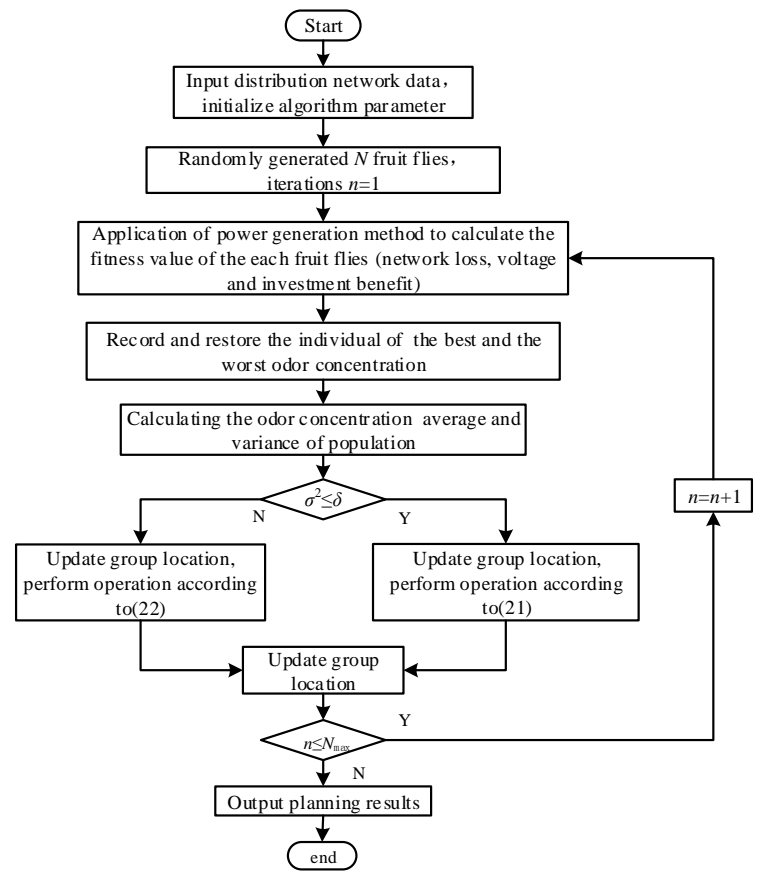

Fig. 1. Process of DG multi objective programming based on MFOA.

\section{Analysis of Cases}

The IEEE-33 node distribution network system is selected as simulation example [7], it's network topology is shown in Fig. 2, and it's total active load and the total reactive load are 3715kW and $2300 \mathrm{kvar}$ respectively, and the reference voltage is $12.66 \mathrm{kV}$. 


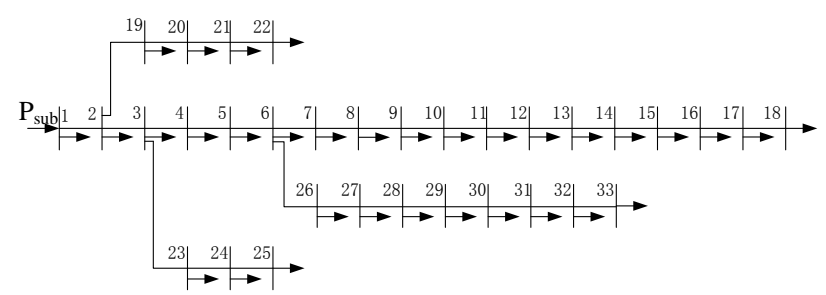

Fig. 2. Diagram of IEEE-33 node system distribution.

DG including wind turbine (WT), photovoltaic (PT), micro fuel stream turbine (MT) and fuel cell (FC), connected into the distribution network is regarded as negative PQ node. The details of DG investment operation parameters is shown in Table 1. The position for MT, PV, FC and WT to be stalled are node 13, 18, 21 and 33 respectively. It is assumed that the power factor of 4 DG types are all 0.9 constantly, and the unit installed capacity is $10 \mathrm{~kW}$. The total output power of DG is less than $30 \%$ of the system load. Algorithm parameters are that the population size of fruit fly (Sizepop) is 50, the maximum number of iterations $\left(T_{\max }\right)$ is 200 , the random initialize interval of the fruit fly group location is $[0,100]$ and the random searching direction and distance of individual is $[-10,10]$.

Table 1. Investment operation data of DG

\begin{tabular}{cccccc}
\hline Type & $\begin{array}{c}\text { Investment cost } \\
\left(10^{4} \mathrm{RMB} / \mathrm{kW}\right)\end{array}$ & $\begin{array}{c}\text { Operating and maintenance } \\
\text { costs }(\mathrm{RMB} / \mathrm{kW})\end{array}$ & Convert coefficient & $\begin{array}{c}\text { capacity } \\
\text { coefficient }\end{array}$ & $\begin{array}{c}\text { government subsidies } \\
\text { (RMB/kW.h) }\end{array}$ \\
\hline WT & 1.3 & 0.032 & 0.1006 & 0.35 & 0.25 \\
PV & 4.55 & 0.013 & 0.0843 & 0.29 & 0.25 \\
FC & 1.82 & 0.320 & 0.9748 & 1.00 & 0 \\
MT & 0.97 & 0.195 & 0.1006 & 1.00 & 0 \\
\hline
\end{tabular}

The paper verifies the validity of MFOA proposed in solving this kind of problem through comparing the convergence curves from the other two intelligence algorithms, FOA and PSO in Fig. 3. It shows that the convergence rate of PSO is same as MFOA, which both converge at 60th iteration, however, the solving accuracy of it is far less than that of MFOA. The convergence rate of FOA is the worst that converges at around 150th iteration, while the solution accuracy of it is higher than PSO, but still lower than MFOA. Consequently, MFOA is better than FOA and PSO in the respects of the convergence rate and the solution accuracy, furthermore, the feasibility of using MFOA to execute the DG optimal configuration calculation is verified.

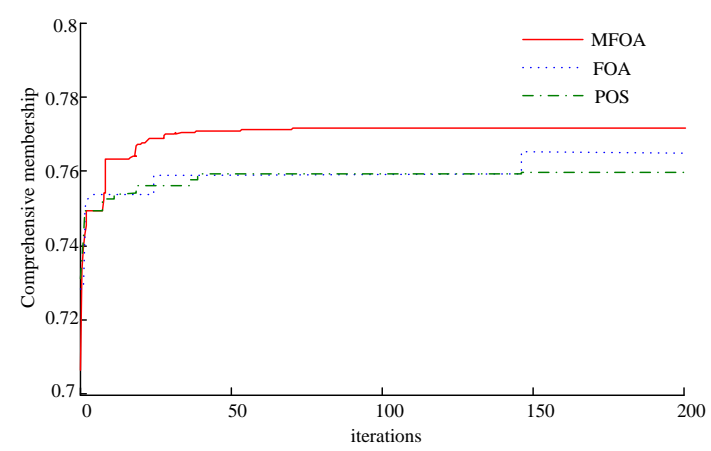

Fig. 3. Convergence curve of different optimization method.

The results of DG optimal configuration solved by different optimization methods are showed in Table 
2. Table 1 shows that MFOA performs excellent effect in the aspect of reducing power loss and voltage index that decreases $57.5 \%$ and $41.6 \%$ separately compared with the data before optimization, though it is the worst in investment efficiency. From analysis in the Fig. 3, we can see that the comprehensive membership of MFOA is better than the rests thought the 3 sub-goals are not the best. Meanwhile the improvement of the voltage quality and the power loss of power distribution network are at the expense of reducing investment interests of DG, and there is a competition between 3 sub-goals. Therefore, the importance degree of every index should be measured comprehensively during the process of DG optimal configuration. The multi-objective model established in4 this paper can reasonably balance the relationships of each sub-goals and reflect the merits of each planning scheme more intuitively.

Table 2. Optimal allocation of DG with different method

\begin{tabular}{|c|c|c|c|c|}
\hline Algorithm & DG's location and contribution $(\mathrm{kW})$ & $C_{\mathrm{psc}}\left(10^{4} \mathrm{RMB}\right)$ & $P_{\text {loss }}(\mathrm{kW})$ & $U($ p.u $)$ \\
\hline Un-optimize & $\begin{array}{c}--- \\
\end{array}$ & $\begin{array}{c}--- \\
\end{array}$ & 224.95 & 1.9080 \\
\hline MFOA & $\begin{array}{l}13-250,18-280 \\
21-200,33-300\end{array}$ & 0.8574 & 95.7159 & 1.9080 \\
\hline FOA & $\begin{array}{c}13-260,18-230 \\
21-90,33-280\end{array}$ & 0.9231 & 102.0374 & 1.1145 \\
\hline PSO & $\begin{array}{l}13-270,18-240 \\
21-110,33-300\end{array}$ & 0.9177 & 97.9214 & 1.1459 \\
\hline
\end{tabular}

The voltage curves from different optimization algorithms are shown in Fig. 4. Fig. 3 illustrates that the voltage curves of all kinds of algorithms performs better significantly, and of all the curve of MFOA is most ideal. The minimum and the average voltage of the system increase from 0.9038p.u and 0.9454p.u to 0.9360 p.u. and 0.9718 p.u separately after optimization with the growth of 0.032 p.u and 0.0264 p.u respectively. To sum up, suitable optimal configuration of DG can lower the transmission losses and improve the quality of voltage as well as the reliability of distribution network.

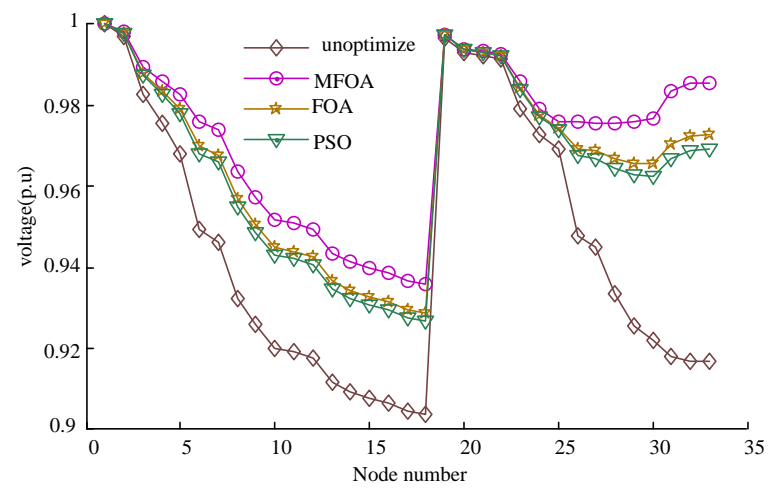

Fig. 4. Voltage curve of different optimization method.

\section{Conclusions}

- The feasibility of DG optimal configuration is evaluated from aspects of economic efficiency, power loss and voltage quality to make the planning the scheme more convincing. And the over-optimized problem due to the different dimensions of each sub-goal can be overcome by using fuzzy modeling technology to give deciders a more intuitive understanding about internal connection among each subgoals.

- MFOA with the introduction of the attractive and the exclusive operation bases on the information of current environment to adjust group location dynamically, which improves the population diversity 
and lowers the risk of falling into the situation of local optimum effectively.

- This article mainly research on the analysis of optimal configuration of DG from the angel of a new intelligence configuration. And the further study will focus on establishing a multi-objective optimization of uncertainty mathematic model considering the random uncertainty brought by DG and using multi-objective optimization algorithm for problem solving.

\section{References}

[1] Guo J, Li X, Zheng W, et al. Comprehensive optimal allocation of intermittent distributed generation power compensation based on bi-level planning. Proceedings of the CSEE, 2013; 33(28):25-33.

[2] Meng X, Gao J, Sheng W, et al. A day-ahead two-stage optimal scheduling model for distribution network containing distributed. Power System Technology, 2015; 39(5):1294-1300.

[3] Zhang L, Tang W, Wang S, el al. Distributed generators planning considering benefits for distribution power company and independent power suppliers. Automation of Electric Power Systems, 2011; 35(4):23-28.

[4] Chakravotry M, Das D. Voltage stability analysis of radial distribution networks. Electrical Power and Energy Systems, 2001; 23:129-135.

[5] Pan WT. A new fruit fly optimization algorithm: taking the financial distress model as an example. Knowledge-Based Systems, 2012; 26:69-74.

[6] Cho JH, Park JI, Jeong JS, et al. Bacterial foraging with quorum sensing based optimization algorithm. Fuzzy Systems, IEEE, 2009:29-3.

[7] Aman MM, Jasmon GB, Bakar AHA, et al. A new approach for optimum simultaneous multi-DG distributed generation Units placement and sizing based on maximization of system load ability using HPSO (hybrid particle swarm optimization) algorithm. Energy, 2014; (66):202-215. 